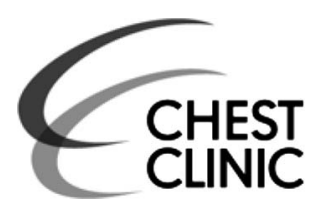
CLIEST

1 Department of Mother and Child Health, Azienda Ospedaliero-Universitaria Ospedali Riuniti, Ancona, Italy ${ }^{2}$ Department of Radiological Sciences, Azienda OspedalieroUniversitaria Ospedali Riuniti, Ancona, Italy

\section{Correspondence to}

Professor Fernando Maria de Benedictis, Division of Pediatrics, Salesi Children's Hospital, 11, via Corridoni, Ancona I-60123, Italy; debenedictis@ospedaliriuniti. marche.it

Received 2 July 2014 Accepted 25 August 2014 Published Online First 5 September 2014

\title{
An adolescent with persistent cough, abdominal pain and refusal to walk from Salesi Children's Hospital
}

\author{
Silvia Angeloni, ${ }^{1}$ Ines Carloni, ${ }^{1}$ Patrizia Osimani, ${ }^{1}$ Lucia Amici, ${ }^{2}$ Cecilia Lanza, ${ }^{2}$ \\ Fernando Maria de Benedictis ${ }^{1}$
}

\section{SA (RESIDENT)}

A 12-year-old boy was admitted to our unit for a 3-day history of abdominal and right groin pain, refusal to walk and relapse of fever. His clinical history was uneventful until 2 weeks before, when he developed acute onset of fever, cough and breathlessness. Right upper lobe pneumonia was diagnosed by chest X-ray and intramuscular ceftriaxone treatment was administered for 10 days followed by fever reduction after 3-day therapy. On admission, he appeared quite unwell with fever and wet cough. He was moderately tachycardic and tachypnoeic with oxygen saturations of $94 \%$ in air. Chest examination revealed dullness to percussion and reduced breath sound over the upper right lung field with a few crackles. Abdomen was diffusely painful and tender to palpation in the left upper quadrant. Mild splenomegaly was also evident. The proximal portion of the right thigh appeared moderately swollen and warm, with no skin colour changes. Laboratory investigation revealed $23000 / \mathrm{mm}^{3}$ white blood cells $(77 \%$ neutrophils), with normal platelet count and haemoglobin level. Erythrocyte sedimentation rate and $\mathrm{C}$ reactive protein were $48 \mathrm{~mm} / \mathrm{h}$ and $7.4 \mathrm{mg} / \mathrm{dL}$ $(<0.5)$, respectively. Liver and renal function tests were normal. Repeated chest X-rays revealed right upper lobe consolidation.

\section{FMDB (SENIOR CLINICIAN)}

We have a previously healthy young boy with persistence of clinical and radiological findings of pneumonia who developed non-respiratory symptoms over the last few days. Correct drug administration, antibiotic resistance, aggravating or underlying conditions, complicated pneumonia and aetiological agents unresponsive to treatment should be taken into account in similar cases. Streptococcus pneumoniae is the most common bacterial cause of community-acquired pneumonia in childhood. Although amoxicillin is recommended as first choice drug for antibiotic therapy, administration of cephalosporins may represent an alternative. ${ }^{1}$ The patient received correct ceftriaxone dosage, the duration of treatment was adequate, and pneumococcal $\beta$-lactam non-susceptibility is rare in Italy. The personal history was not suggestive of either aggravating conditions such as immunodeficiency or underlying lung disease such as cystic fibrosis or immotile cilia dyskinesia. Empyema, necrotising pneumonia and lung abscess represent possible complications of pneumonia, but they can be reasonably excluded by chest X-ray. The role of other aetiological agents such as Mycoplasma pneumoniae and virus should be considered and proper investigation be performed.

\section{PO (INFECTIVOLOGIST)}

Blood cultures were negative. Real-time PCR analysis on blood sample for $S$ pneumoniae and on nasopharyngeal samples for 10 respiratory viruses was also negative. M pneumoniae serology revealed negative immunoglobulin $\mathrm{M}(\operatorname{IgM})$ and positive IgG value that was suggestive of past infection. Cold agglutinins were positive $(1: 512)$ across the thermal range of $4^{\circ} \mathrm{C}-20^{\circ} \mathrm{C}$ and negative at $37^{\circ} \mathrm{C}$.

\section{FMDB}

These laboratory data do not exclude a recent M pneumoniae infection. Indeed, high titres of cold (IgM)-agglutinins may interfere with determination of IgM antibodies against $M$ pneumoniae at standard assessment and lead to false-negative results. Blood incubation at $37^{\circ} \mathrm{C}$ and determination of IgA antibodies against $\mathrm{M}$ pneumoniae are both necessary to avoid misinterpretation of data.

\section{IC (STAFF DOCTOR)}

IgM against $M$ pneumoniae raised to $236 \mathrm{U} / \mathrm{mL}$ after blood incubation at $37^{\circ} \mathrm{C}$ and specific IgA antibodies were positive $(>100 \mathrm{U} / \mathrm{mL})$, thus suggesting a recent $M$ pneumoniae infection. With regard to non-respiratory symptoms presented by the child, abdominal pain may be justified by pleural involvement and groin pain by hip joint arthritis, as either reactive manifestation or septic complication of pneumonia. Both symptoms are consistent with M pneumoniae infection.

\section{FMDB}

Some children with pneumonia may present with abdominal pain, but it is more common in the early stage and when a lower lobe is involved; this is not our case. Groin pain may be associated with hip joint arthritis, but a swollen thigh does not fit well with such diagnostic hypothesis. Alternative causes, such as adenomesenteritis and septic osteomyelitis, should be considered in the differential diagnosis of abdominal and groin pain, respectively. Noninvasive imaging investigation may be helpful to exclude these conditions and to better elucidate the nature of symptoms. 


\section{LA (RADIOLOGIST)}

Right hip joint ultrasound (US) was negative, but US and Doppler colour-flow imaging revealed an extensive thrombus in the superficial right femoral vein, extending from the adductor hiatus distally to common femoral and external iliac vein proximally. Abdominal US revealed mild splenomegaly with heterogeneous echogenicity; colour Doppler showed absence of blood flow in the splenic parenchyma and splenic artery, which was consistent with complete thrombotic obliteration.

\section{FMDB}

M pneumoniae infection is usually responsible for mild respiratory disease, but severe and/or extrapulmonary manifestations including thromboembolism may occur. ${ }^{2}$ A recent review reported a total of 23 cases of arterial and venous thromboses involving several localisations (lower limbs, cerebral, mesenteric, spleen, pulmonary and intracardiac) during $M$ pneumoniae infection. ${ }^{3}$ Most patients were children and had no congenital factors predisposing to thromboses. Transient presence of antiphospholipid antibodies, a heterogeneous group of antibodies directed against plasma proteins bound to phospholipid surfaces, has been well documented in such cases and they may actively contribute to hypercoagulability. ${ }^{4}$ Full thrombophilia screening to evaluate the systemic clotting state and additional imaging investigation to find out possible thromboembolic localisations should be promptly performed.

\section{IC}

The coagulation screen showed normal values for prothrombin time, activated thromboplastin time, antithrombin III; fibrinogen and fibrin D-dimer values were $629 \mathrm{mg} / \mathrm{dL}(\mathrm{nv}<350)$ and $3.912 \mathrm{ng} / \mathrm{mL}$ (nv <230), respectively. The thrombophilia screen revealed positive anticardiolipin (aCL) IgM antibodies $(40 \mathrm{MPL} / \mathrm{mL})$ and negative anti-aCL IgG and anti- $\beta 2$-glycoprotein I IgG and IgM antibodies. Lupus anticoagulant was positive. Protein $C$, protein $S$, homocysteine levels and antinuclear antibodies were normal. The screening for factor $\mathrm{V}$ G1619A Leiden and factor II G20210A mutations was negative.

\section{CL (SENIOR RADIOLOGIST)}

CT of the chest with contrast showed right upper lobe infiltrate with mild pleural effusion. Partial filling defects surrounded by contrast material ('polo mint' sign) in the lower lobar and segmental branches of both pulmonary arteries were also revealed, a finding that is suggestive of bilateral pulmonary thromboembolism (figure 1A). CT of the abdomen revealed complete thrombosis of the splenic artery and complete non-perfusion of the spleen with capsular enhancement (figure 1B) that is suggestive of total splenic infarction; hepatic artery was also partially obstructed by a thrombus (figure 1C). Transthoracic echocardiography and cerebral contrast-enhanced MRI were both normal.

\section{FMDB}

There are no doubts that the child was suffering from multiple arterial and venous thromboembolisms during the course of $M$ pneumoniae infection. He presented with abdominal pain and a swollen thigh: extensive thrombosis of intra-abdominal arteries and femoral veins is consistent with this clinical presentation. Splenic infarction may justify the clinical finding of enlarged spleen at physical examination, as well. Despite pulmonary embolism, our patient did not present significant symptoms in association with tachypnoea and cough; it is conceivable that he remained haemodynamically stable over time, being the pulmonary emboli relatively small. Although a recent systematic review provided insufficient evidence to
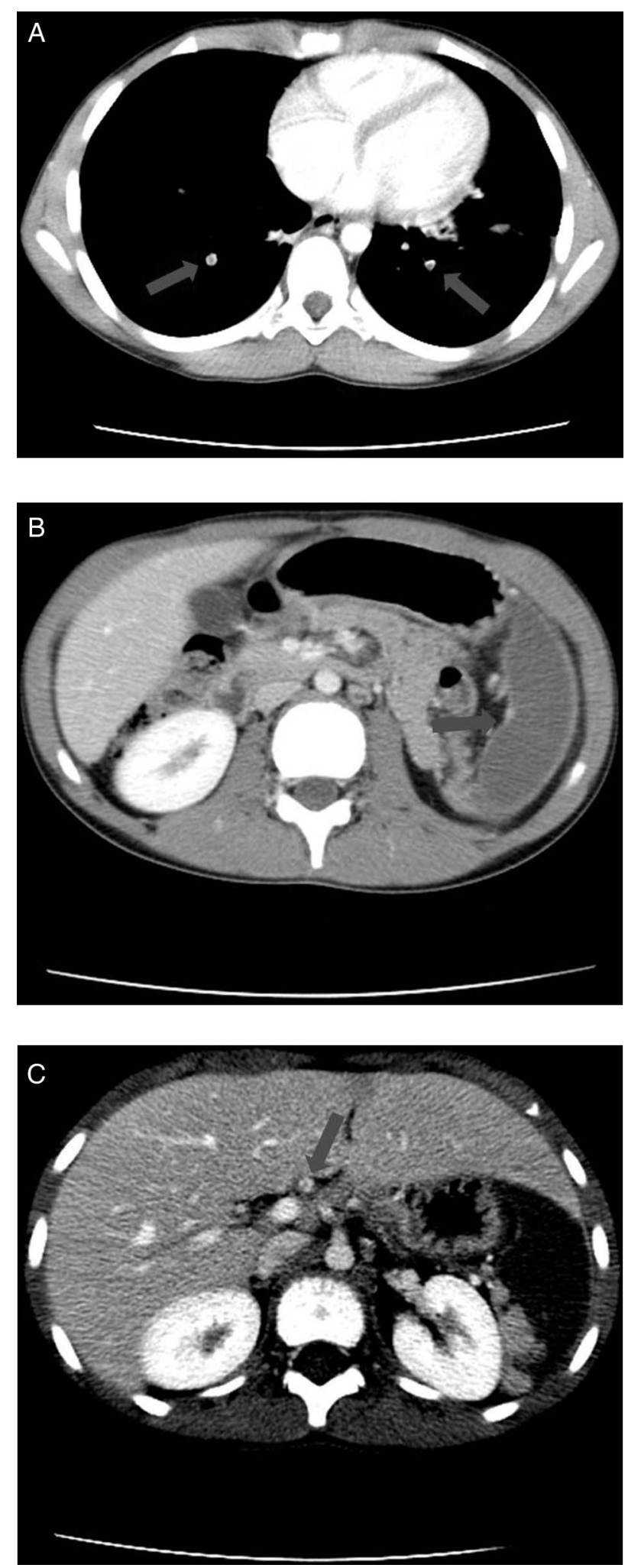

Figure 1 (A) CT of the chest with contrast: partial filling defects surrounded by contrast material ('polo mint' sign) in the segmental branches of both pulmonary arteries. (B) CT of the abdomen with contrast: complete non-perfusion of the spleen with capsular enhancement. (C) CT of the abdomen with contrast: hepatic artery partially obstructed by a thrombus. 
support the efficacy of antibiotics on community-acquired pneumonia due to $M$ pneumoniae, ${ }^{5}$ antibiotic therapy and anticoagulant treatment should be promptly introduced in this case.

\section{IC}

Antimicrobial treatment consisting of oral clarithromycin for 10 days and regular anticoagulation regimen with low molecular weight heparin (enoxaparin, $100 \mathrm{IU} / \mathrm{kg}$ every $12 \mathrm{~h}$ ) for 3 months were scheduled. Progressive clinical improvement was obtained, the patient was discharged at day 20 and no thrombotic or haemorrhagic complications intervened over the anticoagulation treatment period. Repeated screening for circulating anticoagulants was negative at 4 weeks, thus confirming that prothrombotic state was not due to an underlying inherited thrombophilia. Doppler examination showed complete resolution of thrombosis of femoral veins, pulmonary arteries and hepatic artery over a 3-week period; however, splenic artery obliteration persisted and spleen fibrotic changes developed eventually. Due to functional asplenia, proper immunisation against encapsulated organisms, including pneumococcal conjugate vaccine 13 and meningococcal conjugate vaccine 4, was provided.

\section{FMDB}

Thromboembolism is one of the extrapulmonary manifestations of $M$ pneumoniae infection, which also include dermatological, cardiovascular, gastrointestinal, haematological, neurological, musculoskeletal, sensory organ and urogenital tract involvement. These manifestations can occasionally coexist. The mechanism by which $M$ pneumoniae infection can cause thrombosis may be multifactorial including the production of locally induced cytokines, the production of autoantibodies and a vascular-occlusive mechanism with or without systemic thrombophilic state. ${ }^{6}$ These mechanisms are by no means mutually exclusive and may interact. To the best of our knowledge, there are several clinical and radiological aspects that have never been described so far, and that makes our case unique: concomitant thromboembolic involvement of arterial and venous vessels (isolated arterial or venous involvement has been reported); complete infarction of spleen (only peripheral wedge-shaped lesions have been reported) and the bilateral pulmonary embolism (only unilateral pulmonary artery involvement has been described). ${ }^{7}$

Contributors SA treated the patient initially and contributed to the manuscript. IC treated the patient and conceived the manuscript. PO treated the patient and contributed to the interpretation of laboratory data. LA performed all radiological images and contributed to the radiological aspects of discussion. $\mathrm{CL}$ contributed to the imaging interpretation and discussion. FMdB supervised the treatment of the patient, contributed to the clinical aspects of discussion and critically reviewed the manuscript.

\section{Competing interests None.}

\section{Patient consent Obtained.}

Provenance and peer review Not commissioned; internally peer reviewed.

\section{REFERENCES}

1 Harris M, Clark J, Coote N, et al. British Thoracic Society guidelines for the management of community acquired pneumonia in children: update 2011. Thorax 2011;66:ii1-ii23.

2 Waites KB, Talkington DF. Mycoplasma pneumonia and its role as human pathogen. Clin Microbiol Rev 2004;17:697-728.

3 Flateau C, Asfalou I, Deman AL, et al. Aortic thrombus and multiple embolisms during a Mycoplasma pneumoniae infection. Infection 2013;41:867-73.

4 Snowden N, Wilson PB, Longson M, et al. Antiphospholipid antibodies and Mycoplasma pneumoniae infection. Postgrad Med J 1990;66:356-62.

5 Biondi E, McCulloh R, Alverson B, et al. Treatment of mycoplasma pneumonia: a systematic review. Pediatrics 2014;133:1081-90.

6 Narita M. Pathogenesis of extrapulmonary manifestations of Mycoplasma pneumonia infection with special reference to pneumonia. J Infect Chemother 2010;16:162-69.

7 Brown SMN, Padley S, Bush A, et al. Mycoplasma pneumonia and pulmonary embolism in a child due to acquired prothrombotic factors. Pediatr Pulmonol 2008;43:200-2. 